\title{
Fast Kinematic Limit Analysis of FRP-Reinforced Masonry Vaults. I: General Genetic Algorithm-NURBS-Based Formulation
}

\author{
Andrea Chiozzi ${ }^{1}$; Gabriele Milani ${ }^{2}$; and Antonio Tralli ${ }^{3}$
}

\begin{abstract}
A new approach for the limit analysis of masonry vaults retrofitted with fiber-reinforced polymers (FRP) based on an upper bound formulation is presented in this paper. In particular, a new genetic algorithm (GA)-nonuniform rational $b$-spline (NURBS)-based general framework for the limit analysis of curved masonry structures tailored upon an upper bound formulation is discussed thoroughly in the present Part I. A given FRP-reinforced masonry vault can be geometrically represented by a NURBS parametric surface, and a NURBS mesh of the given surface can be generated. Each element of the mesh is a NURBS surface itself and can be idealized as a rigid body. An upper bound limit analysis formulation, which takes into account the main characteristics of masonry material and FRP reinforcement, is deduced, with internal dissipation allowed exclusively along element interfaces. The approach is capable of well predicting the load-bearing capacity of any reinforced masonry vault of arbitrary shape, provided that the initial mesh is adaptively adjusted by means of a metaheuristic approach (i.e., a suitable GA) to enforce that element edges accurately approximate the actual failure mechanism. The approach is validated and discussed in Part II, which is devoted to presenting a number of structural analyses of FRP-reinforced vaults. DOI: 10.1061/(ASCE) EM.1943-7889.0001267. () 2017 American Society of Civil Engineers.
\end{abstract}

\section{Introduction}

As seen in recent seismic events, the inadequate performance of masonry constructions in particular historical buildings under earthquake actions is a critical issue. Traditional retrofitting techniques, such as the use of external steel plates or reinforced concrete coatings, have been shown to be uneconomical, inefficient, and often nonviable. Moreover, they present significant shortcomings, mainly because of the additional mass put on the structure. For these reasons, in the last decades, the use of fiber-reinforced polymer (FRP) strips for retrofitting masonry constructions has become more and more attractive. In fact, this is a very light solution, which provides both high mechanical strength and resistance to corrosion, and is available in a wide set of different commercial forms like laminates, textiles, and tendons (Corradi et al. 2002; Korany et al. 2001). Most common structural units, such as planar walls or curved arches and vaults, can be suitably retrofitted through the application of composite materials on their surfaces (both intrados and extrados), thus preventing or modifying the original failure mechanisms and therefore increasing the overall bearing capacity. Even if FRP-based retrofitting techniques are currently quite popular, from a computational viewpoint, they pose very complex mechanical issues, mainly because of brittle phenomena involving both masonry material and FRP-to-masonry adhesive

${ }^{1}$ Postdoctoral Fellow, Dept. of Engineering, Univ. of Ferrara, Via Saragat 1, 44122 Ferrara, Italy (corresponding author). E-mail: andrea .chiozzi@unife.it

${ }^{2}$ Associate Professor, Dept. of Architecture, Built Environment and Construction Engineering, Technical Univ. of Milan, Piazza Leonardo da Vinci 32, 20133 Milan, Italy. E-mail: gabriele.milani@polimi.it

${ }^{3}$ Full Professor, Dept. of Engineering, Univ. of Ferrara, Via Saragat 1, 44122 Ferrara, Italy. E-mail: tra@unife.it

Note. This manuscript was submitted on July 11, 2016; approved on January 25, 2017No Epub Date. Discussion period open until 0, 0; separate discussions must be submitted for individual papers. This paper is part of the Journal of Engineering Mechanics, (C) ASCE, ISSN 0733-9399. bonding, which makes the prediction of the behavior of FRPreinforced structures a challenging task. This fact becomes even more remarkable when handling curved masonry structural elements, in which the entangled interdependence of in- and out-ofplane actions makes the problem even more complex. Up until 15 years ago, research was mainly focused on the mechanics of FRP-retrofitted reinforced concrete elements (Saadatmanesh 1994). In the last decade, a considerable body of work, both theoretical and experimental, has addressed FRP application to masonry as well (Aiello and Sciolti 2006; Cancelliere et al. 2010; Fagone and Briccoli Bati 2008; Fedele and Milani 2011; Ghiassi et al. 2012; Grande et al. 2008; Luciano and Sacco 1998; Marfia and Sacco 2001; Oliveira et al. 2011; Valluzzi et al. 2001). Furthermore, the scientific community has recently put considerable effort into devising simple mathematical relations that allow practitioners to correctly tackle the design of FRP-strengthened masonry structures [e.g., Triantafillou (1998), Triantafillou and Fardis (1997)]. Finally, Italian guidelines (CNR-DT200 2013) proposed a simplified but sound interpretation of the phenomenon of delamination.

Masonry is ubiquitous among historical buildings and monumental constructions (Chiozzi et al. 2016d; Como 2013). In particular, masonry vaults constitute one of the prevailing structural types in the historical built environment worldwide. Therefore, the interest in safeguarding them and the necessity of new computational tools for their structural assessment, even in the presence of FRP reinforcements, is increasing over time. As pointed out in Como (2013) and Huerta (2008), the modern theory of limit analysis, which has been specialized to the case of masonry structures by Heyman [e.g., Heyman (1997)], is one of the most reliable tools for evaluating the load-bearing capacity of masonry vaults.

Recently developed computational methods for masonry vaults can be classified into two wide categories: the finite-element (FE) methods developed both for nonlinear incremental analysis (Milani and Tralli 2012) and for limit analysis (Milani et al. 2008), and the thrust-network methods (Block et al. 2006; Block and Lachauer 2013) directly based on a lower bound formulation 
(Angelillo et al. 2013). Practical application of these methods requires skilled users and, in the case of thrust-network methods, the definition of an equilibrium surface for the vault, which is a priori unknown.

From a technical viewpoint, the limit analysis FE procedures are mainly based on the upper bound theorem (kinematic approach). For cohesive frictional materials, like masonry, it has been shown that the solution is much more reliable and physically sound when dissipation is also admitted along interfaces between contiguous elements (Sloan and Kleeman 1995; Tralli et al. 2014). It should be pointed out that the well-established lower and upper bound theorems of limit analysis enable rigorous determination of an arbitrary small interval to which the exact collapse load of a given perfectly plastic structure belongs. Nevertheless, when such theorems are used in conjunction with a FE procedure, the ability to find a small enough interval (i.e., a good estimate) for the actual collapse load depends strongly on the efficiency of the particular finite elements used and not only on the efficiency of the algorithm used to solve the resulting optimization problem. Traditional procedures devised for enhancing overall efficiency are based on the use of improved velocity field interpolation schemes within elements, for example, by using higher-degree polynomial expansions (Yu and Tin-Loi 2006). For instance, the so-called free Galerkin method and the p-FEM approach introduced by Chen et al. (2008), Ngo and Tin-Loi (2007), and Tin-Loi and Ngo (2003) were devised following this idea.

Still, higher-order elements exhibit special issues when performing strict upper bound analyses in view of the fact that the flow rule has to hold right through each element, whereas, from a practical viewpoint, this condition can be enforced on a finite number of points only. To bypass this drawback, Sloan and Kleeman (1995) proposed a constant strain element with a discontinuous displacement field. More recently, it has been shown that, provided certain conditions are satisfied, conventional linear strain elements can also be used to compute strict upper bounds for a general convex yield function, even when the displacement field is quadratic discontinuous (Makrodimopoulos and Martin 2008, 2007).

A rather straightforward alternative is to adopt a remeshing technique; see, for instance, Christiansen and Andersen (1999) or Christiansen and Pedersen (2001). Although this technique is quite simple, it presents the noticeable shortcoming of an exponential growth of the computational effort after just a small number of iterations because of the discretization being constantly refined at certain locations.

For all structural problems characterized by complex geometries and internal stress distributions, thus requiring a large number of decision variables, as is the case with masonry vaults, a promising alternative approach is based on the adoption of meshes made of rigid and infinitely resistant elements in which plastic dissipation is permitted only along interfaces between adjoining elements. This choice is also in agreement with the actual behavior at failure of masonry (especially if made of bricks characterized by good strength properties), which exhibits collapse mechanisms characterized by large blocks mutually roto-translating.

From a computational standpoint, whereas the number of variables is hugely reduced, the collapse mechanism is bound to run entirely along interfaces. Therefore, the problem turns out to be highly mesh dependent, with the probable outcome of a considerable error in the evaluation of the load-bearing capacity, which, given the upper bound formulation, is usually overestimated. For this reason, the alignment of interfaces becomes pivotal, and a FE procedure will perform very poorly if an inappropriate mesh is adopted. Aside from adaptive remeshing strategies, which have been proposed in the past to bypass this issue [see Lyamin et al.
(2004, 2005)], an alternative approach in which mesh nodal points are iteratively moved through a sequential linear programming (SLP) scheme has been introduced by Milani (2015) and Milani and Lourenço (2009). Although the procedure was proven quite effective, it still needs 50-100 triangular elements for common problems of technical interest and especially requires the evaluation of node position first derivatives with respect to analytical expressions of the surfaces where the nodes are located. Unfortunately, especially for masonry vaults, the correct representation of geometry is paramount because equilibrium is satisfied only if the thrust surface is entirely contained within the structural thickness of the vault, point-by-point.

Nonuniform rational $b$-spline (NURBS) functions are approximating base functions widely used in the field of three-dimensional (3D) modeling (Piegl and Tiller 1995) for their ability to approximate the actual geometry in an extremely accurate way. Recently, some authors have introduced the idea of using NURBS curves as the basis for the limit analysis of masonry arches through a simple lower bound formulation (Chiozzi et al. 2016a, c). The idea was then extended to unreinforced masonry vaults and walls by using a suitable upper bound formulation in Chiozzi et al. (2017a, c).

In the present paper, a novel NURBS-based approach for the homogenized limit analysis of FRP-reinforced masonry vaults based on the upper bound theorem is proposed. Vault geometry can be described by a NURBS representation of the midsurface, which can be generated within any commercial free-form modeler, together with information about the local thickness at each point of the surface. By exploiting the properties of NURBS functions, a mesh of the given surface made of very few elements, which still provides an exact representation of the vaulted surface, can be obtained. Therefore, a given masonry vault with any geometry can be represented by few NURBS parametric elements. Each element of the mesh is a NURBS surface itself and is idealized as a rigid body.

FRP reinforcement, typically made of long composite strips in adherence with the underlying masonry material, is modeled through NURBS surfaces as well. Like masonry elements, FRP elements are treated as rigid bodies. Such elements are characterized by both FRP-masonry interfaces and FRP-FRP interfaces. In particular, FRP-FRP interfaces are always placed in correspondence with interfaces between reinforced masonry elements, whereas FRP-masonry interfaces are placed along the intrados or extrados of the reinforced masonry element.

Starting from the obtained rigid body assembly, an upper bound limit analysis problem with very few optimization variables can be devised that takes into account the main aspects of masonry material through homogenization (i.e., negligible tensile strength, good compressive strength, and orthotropy at failure because of brick arrangement) and in which dissipation is allowed exclusively along masonry element edges, FRP-FRP, and FRP-masonry interfaces.

Because of the very limited number of rigid elements used, the quality of the collapse load so found depends on the shape and position of the interfaces, where dissipation is allowed. Mesh adjustments are therefore needed, but the utilization of SLP (which would be quite cumbersome in the presence of curved surfaces) can be circumvented by adopting a simple metaheuristic like a standard genetic algorithm (GA) or a GA equipped with nonstandard optimization tools, like zooming and elitist strategies; see Milani and Milani's (2008) approach to mesh adjustment.

In the GA-NURBS approach proposed, each individual forming the population is represented by a mesh, and every iteration requires the solution of a linear programming (LP) problem. As a result of the extremely diminished number of NURBS elements used in the discretization (and hence the number of variables of 
the LP problem), the computational effort required at each iteration is almost negligible. After each generation, the GA governs the evolution of a population of potential failure mechanisms, allowing only the fittest individuals to survive and thus producing better approximations of the solution, i.e., moving the interfaces toward the actual failure mechanism. More precisely, at each generation, a new approximated solution is computed based on the principle of natural selection, in which individuals are selected and ranked according to their level of fitness (i.e., the value of the collapse load), breeding them by means of operators inherited from the field of natural genetics (crossover, reproduction, and mutation). Authors have found this process quickly brings about the evolution of the population toward a set of individuals that are better fit to the surrounding environment than previous generations, with a very accurate estimation of both collapse loads and failure mechanisms after a few generations.

The strength of the proposed GA-NURBS method lies in the fact that even by using a mesh made of very few elements (which therefore require negligible computational time to have an estimate of collapse loads), it is possible to precisely describe the geometry of the vault and therefore obtain accurate load multipliers and failure mechanisms. Therefore, the approach exhibits an edge over existing methods for the collapse analysis of FRP-reinforced masonry vaults in terms of computational efficiency. Furthermore, because NURBS represents a standard in the field of 3D modeling, the proposed method can easily be integrated within existing commercial computer-aided design (CAD) software packages, which are popular in the community of professional engineers and architects, thus allowing for the diffusion of safety assessment of masonry vaults through kinematic limit analysis across a broad professional audience. Finally, this method allows the experienced user to greatly improve both accuracy of results and computational time by choosing an intelligent subdivision of geometry, which can easily be driven by the GA as close as possible to the expected failure mechanism.

The paper is organized into two parts. The present Part I is devoted to discussing in due detail the main aspects of the theoretical framework behind the proposed methodology and is organized as follows: in the "NURBS Geometric Model" section, the description of a reinforced masonry vault through a NURBS surface representation and explains how a NURBS mesh can be defined on it, both for masonry and for FRP reinforcement; in the "Kinematic Limit Analysis" section, an upper bound limit analysis formulation with NURBS rigid elements is proposed based on the NURBS geometric representation of the FRP-reinforced masonry vault, which allows computation of the collapse load for a set of given failure mechanisms; and the last section outlines the GA strategy, which is capable of selecting the correct failure mechanism by adequately adjusting the initial mesh. Part II (Chiozzi et al. 2017b) is devoted to presenting the implementation of the described approach, and the collapse mechanisms of a number of FRP-reinforced curved masonry structures (parabolic arch, skew arch, hemispherical dome, cross vault, and cloister vault) are analyzed and discussed in detail.

\section{NURBS Geometric Model}

FRP-reinforced masonry vaults can be modeled in any free-form 3D modeler using NURBS surfaces for both FRP strips and the underlying masonry structure, which can be adequately described by its mean surface. NURBS basis functions share many similarities with $b$-spline basis functions, to which they are strongly connected. $B$-spline basis functions are piecewise polynomials defined by the so-called knot vector $\Xi=\left\{\xi_{1}, \xi_{2}, \ldots, \xi_{n+p+1}\right\}$, composed of points in the parameter space $\xi_{i} \in[0,1]$, known as knots. In the expression of the knot vector, $p$ denotes the degree of the polynomials, whereas $n$ is the overall number of basis functions. The $i$ th $b$-spline basis function $N_{i, p}$ is obtained through the Cox-de Boor recursion equation (Piegl and Tiller 1995). The NURBS basis functions $R_{i, p}$ are expressed as

$$
R_{i, p}(\xi)=\frac{N_{i, p}(\xi) w_{i}}{\sum_{i=1}^{n} N_{i, p}(\xi) w_{i}}
$$

where $w_{i} \in \mathbb{R}=$ weights. NURBS shares many properties with $b$-spline basis functions. Although $b$-spline basis functions could be regarded as special NURBS basis functions with all weights set equal to one, NURBS basis functions have the notable capability of representing exactly the geometry of a broad class of curves, among which the special case of conic sections should be mentioned (Cottrell et al. 2009), and of the surfaces generated by these curves. Given a set $\mathbf{B}_{i} \in \mathbb{R}^{3}$ of control points, a NURBS curve of degree $p$ is a parametric curve in the 3D Euclidean space defined as

$$
\mathbf{C}(u)=\sum_{i=1}^{n} R_{i, p}(\xi) \mathbf{B}_{i}
$$

Analogously, a NURBS surface of degree $p$ in the $u$-direction and $q$ in the $v$-direction is a parametric surface in the threedimensional Euclidean space defined as

$$
\mathbf{S}(u, v)=\sum_{i=0}^{n} \sum_{j=0}^{m} R_{i, j}(u, v) \mathbf{B}_{i, j}
$$

where $\left\{\mathbf{B}_{i j}\right\}$ forms a bidirectional net of control points. A set of weights $\left\{w_{i, j}\right\}$ and two separate knot vectors in both the $u$ - and $v$-directions must be defined. Given a NURBS surface $\mathbf{S}(u, v)$, isoparametric curves on the surface can be defined by fixing one parameter in the parameter space and letting the other vary. By fixing $u=u_{0}$, the isoparametric curve $\mathbf{S}\left(u_{0}, v\right)$ is defined on the surface $\mathbf{S}$, whereas by fixing $v=v_{0}$, the isoparametric curve $\mathbf{S}\left(u, v_{0}\right)$ is obtained. Many commercial free-form surface modelers, such as Rhinoceros, use NURBS representation and its properties to generate and manipulate surfaces in the $3 \mathrm{D}$ space. In the numerical simulations contained in Part II, both vault midsurfaces and FRP strips have been modeled within Rhinoceros as NURBS surfaces, and the resulting NURBS structure has been imported within a $M A T L A B$ environment through the Initial Graphics Exchange Specification (IGES) standard (USPRO 1996).

Once the NURBS structure created within Rhinoceros has been transferred to the MATLAB environment, it is possible to manipulate it by exploiting NURBS properties to define a NURBS mesh of the masonry midsurface in which each element is a NURBS surface itself. Furthermore, it is possible to model vault thickness at each interface between elements by offsetting the original interface inward and outward through a translation in the direction normal to the NURBS surface. Fig. 1(a) represents an example of a Rhinoceros 3D model of a cross vault reinforced with FRP strips at both the extrados and intrados of the four main arches; both FRP strips and the midsurface of the masonry structure have been modeled as NURBS surfaces. Fig. 1(b) represents the structural mesh obtained within a MATLAB environment after exporting the original NURBS structure through the IGES standard and subdividing the original surface into a set of smaller NURBS elements to which the real vault thickness is assigned. Typically, the easiest way to generate a NURBS mesh on a given surface is to define a subdivision of 


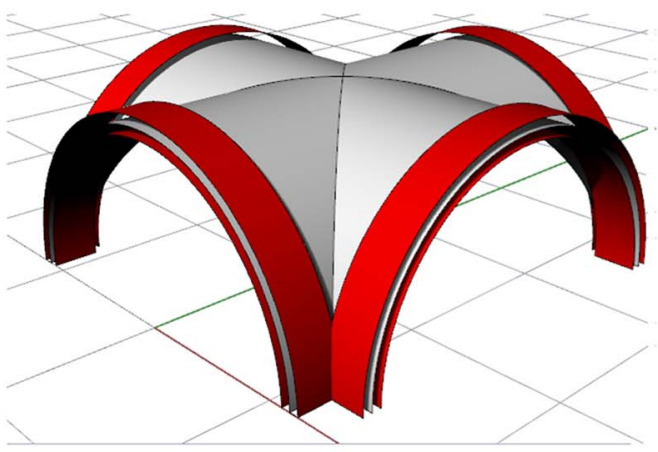

(a)

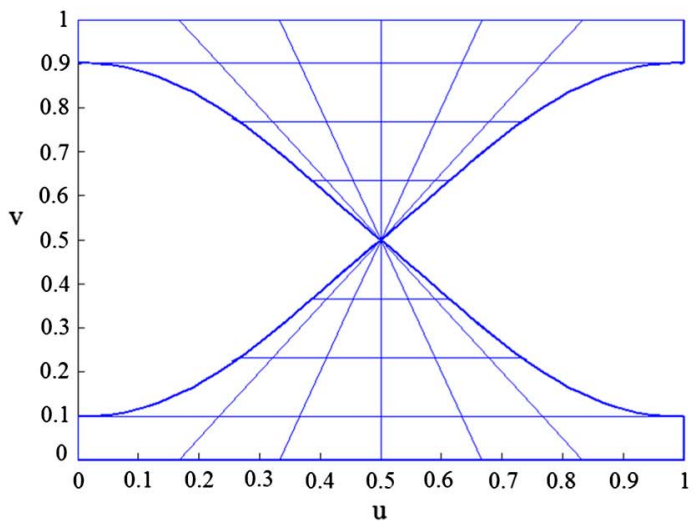

(c)

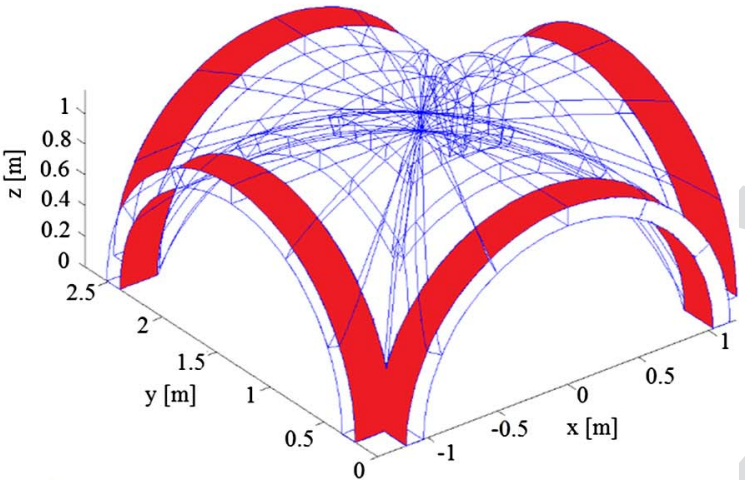

(b)

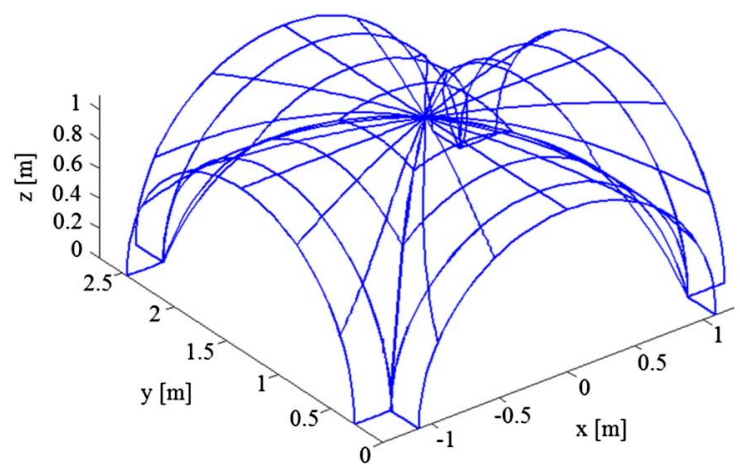

(d)

F1:1 Fig. 1. FRP-reinforced cross vault: (a) 3D NURBS model obtained with Rhinoceros; (b) preliminary mesh obtained with MATLAB through the IGES F1:2 standard; (c) parameter space subdivision; (d) corresponding subdivision of the vault midsurface

the two-dimensional parameter space $u-v$, which follows from subdividing the knot vectors in both the $u$ - and $v$-directions into equal intervals. The resulting mesh is defined by isoparametric curves on the surface in the 3D Euclidean space. Each element of the mesh is a NURBS surface, and its edges are branches of isoparametric curves belonging to the initial surface. More precisely, the counterimage of each element of the mesh is a rectangle $S_{i j}=\left[u_{i}, u_{i+1}\right] \times$ $\left[v_{j}, v_{j+1}\right] \in \mathbb{R}^{2}$ defined in the parameter space. More generally, different meshes of the NURBS surface can be obtained for arbitrary partitions of the parameter space into quadrilateral or triangular domains. Particular attention must be addressed to multiple NURBS surfaces composed by Boolean operations between elementary NURBS patches, which is particularly frequent when dealing with geometries of typical masonry vaults. In that case, the counterimage of the surface in the parameter space is not a rectangle of $\mathbb{R}^{2}$ anymore. As an example, the case of the cross vault represented in Fig. 1 is considered: its surface is generated by the Boolean intersection of two orthogonal barrel vaults, which in turn are generated by the extrusion of a semicircular arch along a straight line normal to the circle plane. Its parameter space is reported in Fig. 1(c), along with the subdivision chosen to get a suitable mesh of the vault midsurface [which is depicted in Fig. 1(d)]. As previously stated, the image of each domain is an element of the mesh, which is a NURBS surface itself. The union of every element of the chosen mesh is equal to the original surface, no matter how coarse the mesh is. For each element of the mesh, $E_{i}$ is its counterimage in the two-dimensional parameter space $u-v$ in the quadrangular domain $K_{i}$.

Therefore, the area of the surface can be computed through the following relation:

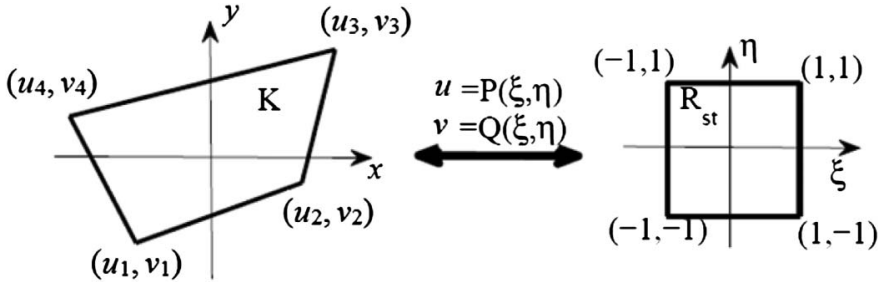

Fig. 2. Linear mapping between $K$ and $R_{s t}$

$$
A_{i}=\iint_{E_{i}} d S=\iint_{K_{i}}\left\|\mathbf{S}_{u} \times \mathbf{S}_{v}\right\| d u d v
$$

where $\mathbf{S}_{u}$ and $\mathbf{S}_{v}=$ partial derivatives of the parametric surface $\mathbf{S}(u, v)$ in the $u$ - and $v$-directions. Analogously, the center of mass of each element may be computed with the following relation:

$$
\mathbf{c}=\frac{1}{A_{i}} \iint_{E_{i}} \mathbf{x} d S=\iint_{K_{i}} \mathbf{S}(u, v)\left\|\mathbf{S}_{u} \times \mathbf{S}_{v}\right\| d u d v
$$

Because the integrals in Eqs. (4) and (5) are evaluated on general quadrangular domains, an isoparametric approach can be adopted for their numerical computation. Let $K$ be a quadrilateral domain in the parameter space with straight boundary lines and vertices $\left(u_{i}, v_{i}\right), i=1,2,3,4$ arranged in counterclockwise order (Fig. 2). The idea is simple: first transform the quadrilateral domain $K$ to the standard quadrilateral element $R_{s t}$ and then apply the Gaussian quadrature. The transformation can be done by using the following nodal shape functions for quadrilaterals: 


$$
\begin{array}{ll}
N_{1}(\xi, \eta)=\frac{1}{4}(1-\xi)(1-\eta), & N_{2}(\xi, \eta)=\frac{1}{4}(1+\xi)(1-\eta), \\
N_{3}(\xi, \eta)=\frac{1}{4}(1+\xi)(1+\eta), & N_{4}(\xi, \eta)=\frac{1}{4}(1-\xi)(1+\eta),
\end{array}
$$

$N_{i}(\xi, n)=1$ at node $i$ and zero at other nodes. Now, it is necessary to construct a linear mapping to map the quadrilateral domain $K$ to the standard square $R_{s t}=[-1,1] \times[-1,1]$ in the auxiliary two-dimensional space $(\xi, \eta)$ (Fig. 2). The mapping can be achieved conveniently by using the nodal shape function as follows:

$$
\begin{aligned}
& u=P(\xi, \eta)=\sum_{i=1}^{4} u_{i} N_{i}(\xi, \eta) \\
& v=Q(\xi, \eta)=\sum_{i=1}^{4} v_{i} N_{i}(\xi, \eta)
\end{aligned}
$$

Then, a given integral over $K$ can be rewritten in the following way as an integral over $R_{s t}$ :

$$
\iint_{K} F(u, v) d u d v=\iint_{R_{s t}} F[P(\xi, \eta), Q(\xi, \eta)]|J(\xi, \eta)| d \xi d \eta
$$

where $J(\xi, \eta)=$ Jacobian of the transformation in Eq. (7).

Therefore, it is now possible to apply the Gaussian quadrature rule for standard square domains

$$
\iint_{K} F(u, v) d u d v=\sum_{i=1}^{N} \sum_{j=1}^{N} w_{i} w_{j} F\left[P\left(\xi_{i}, \eta_{j}\right), Q\left(\xi_{i}, \eta_{j}\right)\right]\left|J\left(\xi_{i}, \eta_{j}\right)\right|
$$

where $\left(\xi_{i}, \eta_{j}\right)$ and $w_{j}=$ Gaussian quadrature points and weights, respectively. In the numerical examples discussed in Part II, a three-point Gauss rule has been adopted in each direction for computing the area [Eq. (4)] and center of mass [Eq. (5)] integrals because this choice provides the needed accuracy. Fig. 3 reports how fast numerical evaluated area integrals [Eq. (4)] converge to the exact value by increasing the number of Gauss points in each direction. In fact, a three-point-per-direction Gauss rule is proven to be sufficiently accurate for the scope of this paper. Finally, two more definitions are needed to apply limit analysis to the obtained assembly of NURBS elements. Given that the NURBS surface

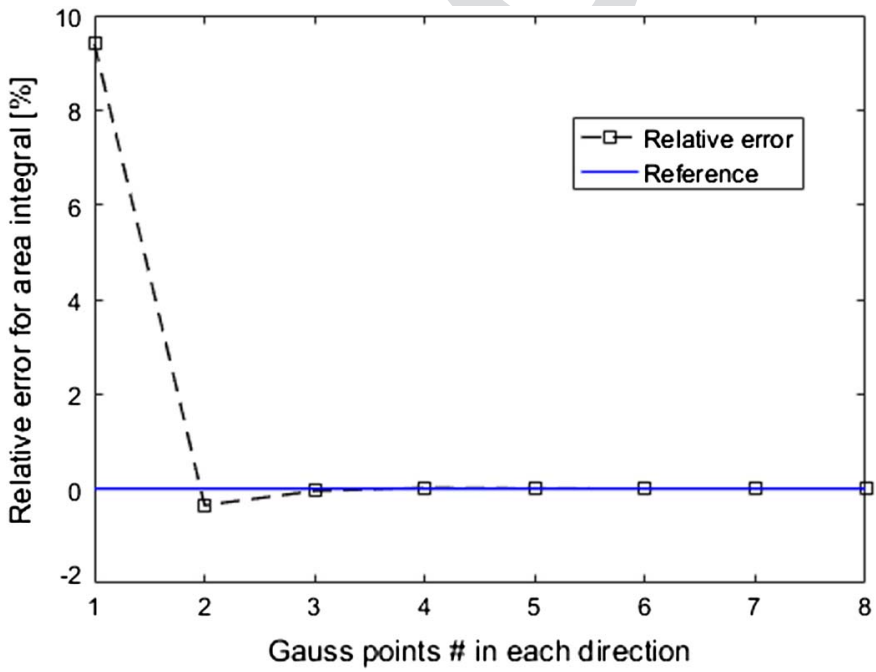

F3:1 Fig. 3. Numerical integration convergence graph with increasing F3:2 Gauss point numbers
$\mathbf{S}(u, v)$ has, in each point, a regular parametrization-i.e., partial derivative vectors $\mathbf{S}_{u}$ and $\mathbf{S}_{v}$ are linearly independent for each couple of parameters $(u, v)$ - the tangent plane is the affine plane in $\mathbb{R}^{3}$ spanned by these vectors and passing through the point $\mathbf{S}(u, v)$.

\section{Kinematic Limit Analysis}

As discussed in the previous section, given the NURBS 3D model of the FRP-reinforced vaulted surface, a NURBS mesh can be defined on the same surface, and a set of rigid elements can be defined for both the masonry structure and the FRP reinforcement. Starting from the geometrical properties of each element, an upper bound formulation of limit analysis can be outlined. Because elements are considered rigid, internal dissipation is permitted only at the interfaces between contiguous elements in the proposed model. This assumption has shown to be sufficiently reliable even for the case of masonry shells subjected to out-of-plane loads and reinforced with FRP strips (Milani 2009). This section summarizes the proposed upper bound formulation.

Let $N_{E}$ be the number of elements composing the NURBS mesh, which geometrically represents the FRP-reinforced vaulted surface. Because each element is considered rigid, the kinematics of each element are determined by the six (three translational and three rotational) generalized velocity components $\left\{u_{x}^{i}, u_{y}^{i}, u_{z}^{i}, \Phi_{x}^{i}, \Phi_{y}^{i}, \Phi_{z}^{i}\right\}$ of its center of mass $G_{i}$, expressed in a global reference system $O x y z$. Dead loads $\mathbf{F}_{0}$ and live loads $\boldsymbol{\Gamma}$ are acting on the structure. Internal dissipation is allowed along interfaces only. Three types of interfaces can be recognized: masonry-masonry, FRP-masonry, and FRP-FRP. Indicating the total number of interfaces by $N_{I}^{\mathrm{TOT}}=$ $N_{I}^{M-M}+N_{I}^{M-F}+N_{I}^{F-F}$, the total internal dissipation power $D_{\text {int }}$ is equal to the sum of the power dissipated along each interface $P_{\text {int }}^{i}$, defined in the present section. Furthermore, total internal dissipation power $D_{\text {int }}$ is equal to the sum of the powers of live $(1 \cdot \Gamma)$ and dead $\left(\mathbf{F}_{0}\right)$ loads, indicated as $P_{\boldsymbol{\Gamma}}$ and $P_{\mathbf{F}_{0}}$, respectively

$$
D_{\text {int }}=\sum_{i=1}^{N_{I}} P_{\text {int }}^{i}=P_{\Gamma}+P_{F_{0}}
$$

where $\Gamma=$ load multiplier. The LP problem related to the kinematic formulation of limit analysis consists of an appropriate minimization of the load multiplier $\Gamma$ under the action of suitable constraints, which are described in the following subsections. The vector of unknowns of the LP problem, $\mathbf{X}$, contains the six generalized velocity components for each element and a number of plastic multipliers along each interface, which will be defined in the following subsections.

\section{Geometric Constraints}

Vertices belonging to element-free edges, which do not constitute an element interface, can be subjected to external kinematic constraints by imposing an assigned value for translational or rotational velocities at these points. For each such vertex $V_{j}$, kinematic constraints can be expressed in terms of generalized velocities of the center of mass of the $i$ th element to which they belong. For example, when only translational velocities of a given vertex $V_{j}$ belonging to element $i$ are constrained to zero, the following relation holds as a geometric constraint:

$$
\mathbf{u}_{V_{j}}=\mathbf{u}^{i}+\mathbf{R}\left[\mathbf{x}_{V_{j}}-\mathbf{x}_{G_{i}}\right]=\mathbf{0}
$$

where $\mathbf{u}_{V_{j}}=\left[u_{x}^{V_{j}}, u_{y}^{V_{j}}, u_{z}^{V_{j}}\right]^{T}=$ three translational velocity components of the vertex $V_{j} ; \mathbf{u}^{i}=\left[u_{x}^{i}, u_{y}^{i}, u_{z}^{i}\right]^{T}=$ three (unknown) translational velocity components of the center of mass of element $i$ to whom vertex $V_{j}$ belongs; and $\mathbf{R}=$ rotation matrix 


$$
\mathbf{R}=\left[\begin{array}{ccc}
0 & -\Phi_{z}^{i} & \Phi_{y}^{i} \\
\Phi_{z}^{i} & 0 & -\Phi_{x}^{i} \\
-\Phi_{y}^{i} & \Phi_{x}^{i} & 0
\end{array}\right]
$$

whose elements are the (unknown) generalized rotational velocities of the center of mass of element $i$. In general, all linear geometric constraints can be rewritten in the following standard form:

$$
\mathbf{A}_{\text {eq, geom }} \mathbf{X}=\mathbf{b}_{\text {eq, geom }}
$$

where $\mathbf{A}_{\text {eq, geom }}=$ matrix of geometric constraints; and $\mathbf{b}_{\text {eq,geom }}=$ corresponding vector of coefficients.

\section{Compatibility Constraints}

Masonry-masonry and FRP-FRP interfaces between elements are planar surfaces whose height at each point of their midlines corresponds to the local structural thickness of the vault and of the reinforcement, respectively, whereas FRP-masonry interfaces are NURBS surfaces placed at the intrados or extrados of the vaulted surface.

\section{Power Dissipation on Masonry-Masonry Interfaces}

To enforce plastic compatibility along masonry-masonry interfaces and correctly evaluate dissipation power, intrados and extrados edges of each interface have been subdivided into an assigned number $\left(N_{s d}^{M}+1\right)$ of points $P_{i}$ (Fig. 4). On each point $P_{i}$ of a masonrymasonry interface, a local reference system $\left(\mathbf{n}^{M}, \mathbf{s}^{M}, \mathbf{t}^{M}\right)$ has been defined where $\mathbf{n}^{M}$ is the unit vector normal to the interface, $\mathbf{s}^{M}$ is the tangential unit vector in the longitudinal direction, and $\mathbf{t}^{M}$ is the tangential unit vector in the transversal direction. On each point $P_{i}$ of each interface, which separates the two elements $E^{\prime}$ and $E^{\prime \prime}$, the following compatibility equation must hold:

$$
\Delta \tilde{\mathbf{u}}=\dot{\lambda} \frac{\partial f}{\partial \boldsymbol{\sigma}}
$$

where $\boldsymbol{\sigma}=\left[\sigma_{n n}, \sigma_{n s}, \sigma_{n t}\right]=$ stress vector acting on $P_{i}$ in the three local reference directions; $f(\boldsymbol{\sigma})=$ suitable yield function; and $\dot{\lambda}=$ unknown plastic multiplier vector. In Eq. (14), $\Delta \tilde{\mathbf{u}}$ is the representation in the local reference system of the quantity $\Delta \mathbf{u}$ in the global reference system, which is defined as

$$
\Delta \mathbf{u}=\mathbf{u}_{P_{i}}^{\prime}-\mathbf{u}_{P_{i}}^{\prime \prime}
$$

where $\mathbf{u}_{P_{i}}^{i}=$ vector composed by the three translational velocity components of the point $P_{i}$ seen as belonging to element $E^{\prime}$ and $\mathbf{u}_{P_{i}}^{\prime \prime} . \Delta \mathbf{u}$ is related to $\Delta \tilde{\mathbf{u}}$ through the following relation:

$$
\Delta \tilde{\mathbf{u}}=\tilde{R} \Delta \mathbf{u}
$$

where $\tilde{\mathbf{R}}=$ suitable $3 \times 3$ rotation matrix whose rows are, respectively, the components of the three local vectors $\left(\mathbf{n}^{M}, \mathbf{s}^{M}, \mathbf{t}^{M}\right)$ expressed in the global reference system.

The yield surface $f(\boldsymbol{\sigma})$ has been obtained by means of a homogenization procedure based on the so-called method of cells (MoC). This approach was first introduced in Aboudi (2013) for unidirectional composites and recently adapted for in-plane loaded masonry walls in Milani and Taliercio (2015). As detailed in what follows, MoC can also be extended to the case of limit analysis of curved masonry structures (i.e., masonry vaults). The method consists of subdividing a representative elementary volume (REV) into six rectangular subcells, as depicted in Fig. 5. Two sets of strain-rate periodic piecewise differentiable velocity fields, one for normal and one

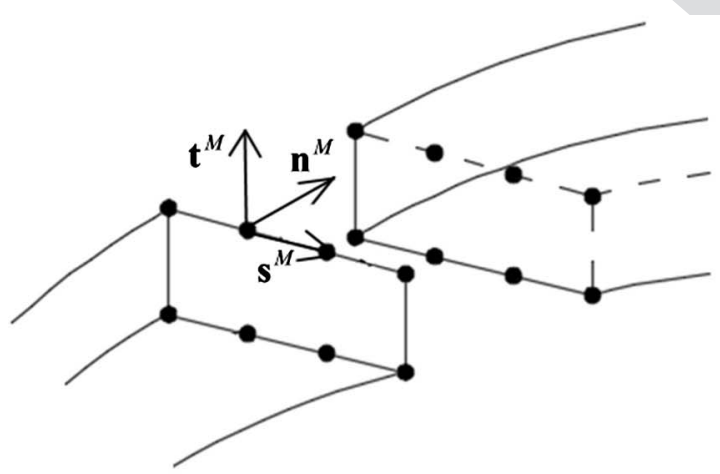

(a)

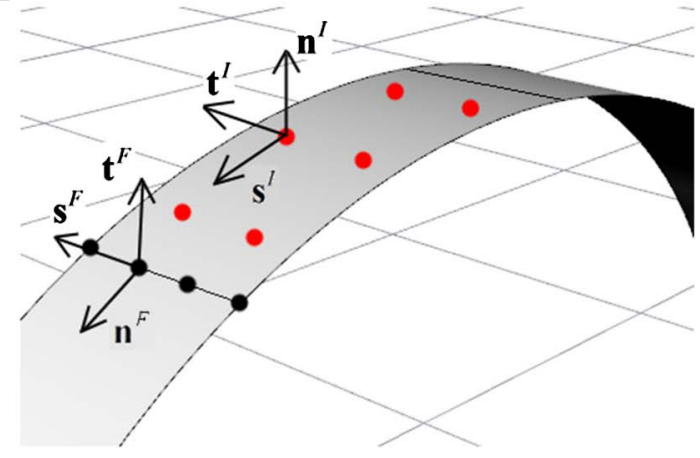

(b)

F4:1 Fig. 4. (a) Masonry-masonry interface and corresponding local reference system; (b) FRP-FRP and FRP-masonry interfaces with corresponding F4:2 local reference system

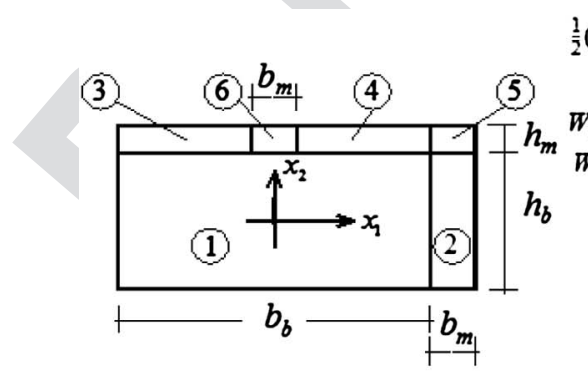

(a)

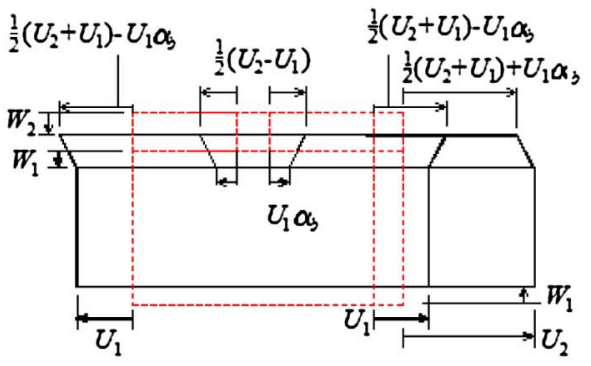

(b)

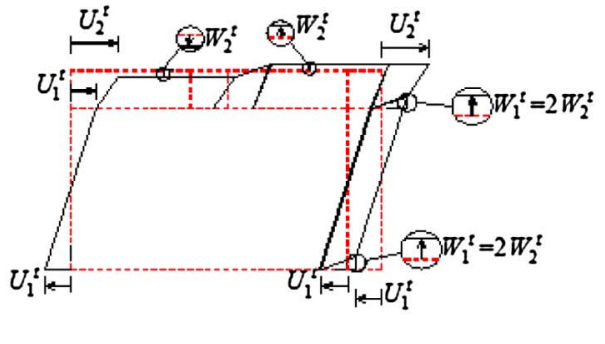

(c)

F5:1 Fig. 5. (a) REV adopted in the MoC approach and subdivision into cells; (b) strain-periodic kinematically admissible velocity field under horizontal or vertical macroscopic normal stresses; (c) strain-periodic kinematically admissible velocity field under macroscopic shear stress 


$$
\begin{aligned}
u_{1}^{n(2)}= & 2 U_{1} \frac{x_{1}}{b_{b}} \quad u_{2}^{n(1)}=-2 W_{1} \frac{x_{2}}{h_{b}} \\
u_{1}^{n(2)}= & U_{1}+\frac{\left(U_{2}-U_{1}\right)\left(x_{1}-\frac{b_{b}}{2}\right)}{b_{m}} \\
u_{2}^{n(2)}= & -2 \frac{x_{2}}{h_{b}}\left[\frac{2\left(W_{1}-W_{2}\right)\left|\frac{b_{m}+b_{b}}{2}-x_{1}\right|}{b_{m}}+W_{2}\right] \\
u_{1}^{n(3)}= & u_{1}^{n(1)}-\frac{\left[U_{1}\left(1+2 \alpha_{b}\right)-U_{2}\right]\left(\frac{h_{b}}{2}-x_{2}\right)}{2 h_{m}} \\
u_{2}^{n(3)}= & -W_{1}+\frac{\left(W_{1}-W_{3}\right)\left(x_{2}-\frac{h_{b}}{2}\right)}{h_{m}} \\
u_{1}^{n(4)}= & u_{1}^{n(1)}+\frac{\left[U_{1}\left(1+2 \alpha_{b}\right)-U_{2}\right]\left(\frac{h_{b}}{2}-x_{2}\right)}{2 h_{m}} \\
u_{1}^{n(5)}= & U_{1}-\frac{\left[U_{1}\left(1+2 \alpha_{b}\right)-U_{2}\right]\left(\frac{b_{b}+b_{m}}{2}-x_{1}\right)\left(x_{2}-\frac{h_{b}}{2}\right)}{b_{m} h_{m}}=u_{2}^{n(3)} \\
& -\frac{\left(U_{1}-U_{2}\right)\left(x_{1}-\frac{b_{b}}{2}\right)}{b_{m}} \\
u_{1}^{n(6)}= & 2 \frac{x_{1}}{b_{b}}\left[U_{1}-\frac{\left.\left(U_{1}+\frac{U_{1}-U_{2}}{2 \alpha_{b}}\right)\left(x_{2}-\frac{h_{b}}{2}\right)\right]}{h_{m}}\right. \\
u_{2}^{n(6)}= & -W_{1}+\frac{\left[W_{2}-W_{3}+2\left(W_{1}-W_{2}\right) \frac{\left|x_{1}\right|}{b_{m}}\right]\left(x_{2}-\frac{\left.h_{b}\right)}{2}\right.}{h_{m}} \\
u_{2}^{n(5)}= & -W_{3} \frac{x_{2}-\frac{h_{b}}{2}}{h_{m}} \\
& \left.-W_{2} \frac{b_{m}}{2}-\left(W_{2}-W_{1}\right)\left|\frac{b_{b}+b_{m}}{2}-x_{1}\right|\right]\left(\frac{h_{b}}{2}+h_{m}-x_{2}\right) \\
u_{m} & \\
&
\end{aligned}
$$

An additional constraint $W_{1}=W_{2}$ is imposed in the model to avoid bilinear terms of the velocity field in cross-joints. Bilinearity makes the check of the associated flow rule inside cross-joints cumbersome, with an experienced negligible modification of the final result. Frames of reference $x_{1}-x_{2}$ and the geometrical meanings of the symbols are provided in Fig. 5(a): brick height $h_{b}$; horizontal bed joint thickness $h_{m}$; ratio $\alpha_{b}$ between $b_{m}$ and $b_{b}$, respectively; vertical bed joint thickness; and brick length. Fields [Eq. (17)] depend on the four degrees of freedom $U_{1}, U_{2}, W_{1}, W_{2}=W_{1}$, and $W_{3}$, with clear physical meaning represented in Fig. 5(b).

Applying a shear deformation mode to the REV, the following velocity fields can be defined on each cell:

$$
\begin{aligned}
& u_{1}^{t(1)}=2 U_{1}^{t} \frac{x_{2}}{h_{b}} u_{2}^{t(1)}=0 \quad u_{1}^{t(2)}=u_{1}^{t(1)} \quad u_{2}^{t(2)}=W_{1}^{t} \frac{x_{1}-\frac{b_{b}}{2}}{b_{m}} \\
& u_{1}^{t(3)}=U_{1}^{t}+\frac{U_{2}^{t}-U_{1}^{t}}{h_{m}}\left(x_{3}-\frac{h_{b}}{2}\right) \quad u_{2}^{t(3)}=-W_{2}^{t} \frac{x_{2}-\frac{h_{b}}{2}}{h_{m}} \\
& u_{1}^{t(4)}=u_{1}^{t(3)} \quad u_{2}^{t(4)}=-u_{2}^{t(3)} \quad u_{1}^{t(5)}=u_{1}^{t(3)} \\
& u_{2}^{t(5)}=-W_{1}^{t} \frac{\left(x_{1}-\frac{b_{b}+b_{m}}{2}\right)\left(x_{2}-\frac{h_{b}}{2}\right)-h_{m}\left(x_{1}-\frac{b_{b}}{2}\right)}{b_{m} h_{m}} \\
& u_{1}^{t(6)}=u_{1}^{t(3)} \quad u_{2}^{t(6)}=W_{1}^{t} \frac{x_{1}\left(x_{2}-\frac{h_{b}}{2}\right)}{b_{m} h_{m}}
\end{aligned}
$$

The symbols $u_{1}^{t(i)}$ and $u_{2}^{t(i)}$ in Eq. (18) indicate vertical and horizontal velocity fields of the $i$ th cell for the shear deformation mode imposed. In Eq. (18), independent variables [degrees of freedom (DOFs)] are represented by $U_{1}^{t}, U_{2}^{t}, W_{1}^{t}$ and $W_{2}^{t}$, whose physical meaning is depicted in Fig. 5(c).

An additional constraint $W_{1}^{t}=2 W_{2}^{t}$ is imposed in the model to make the velocity field compatible between cross-joints and contiguous subcells. Following from the upper bound theorem of limit analysis and assuming the expressions contained in Eqs. (17) and (18) approximate the velocity field upon the representative volume element (RVE), an associated flow rule must hold throughout each subcell. It is now possible to define the horizontal component $u_{1}=u_{1}^{n(i)}+u_{1}^{t(i)}$ and the vertical component $u_{2}=u_{2}^{n(i)}+u_{2}^{t(i)}$ of the field of velocity for the $i$ th subcell. The associated flow rule, which has to hold for each point of a given subcell, can be expressed by three equality constraints as $\dot{\boldsymbol{\varepsilon}}_{p l}^{(i)}=\left[\partial v_{1} / \partial y_{1} \partial v_{2} / \partial y_{2} \partial v_{1} / \partial y_{2}+\right.$ $\left.\partial v_{2} / \partial y_{1}\right]=\dot{\lambda}^{(i)} \partial \mathbf{f}_{b, m} / \partial \boldsymbol{\sigma}$, in which the plastic strain rate field for the considered subcell $\dot{\boldsymbol{\varepsilon}}_{p l}^{(i)}$, the (positive) rate of the plastic multiplier $\dot{\lambda}^{(i)}$, and the failure surface for either brick or mortar $\mathbf{f}^{b, m}$ have been implicitly defined.

If $m$ planes are chosen to approximate the brick and mortar failure surfaces, a set of linear equations in the form $\mathbf{f}_{b, m} \equiv \mathbf{A}^{\text {in }} \boldsymbol{\sigma} \leq \mathbf{b}^{\text {in }}$ is sufficient to define each given failure criterion. Because $\dot{\boldsymbol{\varepsilon}}_{p l}^{(i)}$ is at most linear throughout the subcell, it is possible to enforce plastic admissibility by checking the related condition at three corners only. Therefore, defining $\mathbf{U}=\left\{U_{1}, U_{2}, W_{1}, W_{2}, U_{1}^{t}, U_{2}^{t}, W_{1}^{t}\right\}^{T}$ as the vector containing all seven DOFs representing the microscopic field of velocity, for each subcell, it is possible to introduce nine linear equality constraints in the matrix form $\mathbf{A}_{U(i)}^{\mathrm{eq}} \mathbf{U}+\mathbf{A}_{\lambda(i)}^{\mathrm{eq}} \dot{\boldsymbol{\lambda}}^{(i)}=\mathbf{0}$, where $\dot{\lambda}^{(i)}=\left[\begin{array}{lll}\dot{\lambda}_{A}^{(i) T} & \dot{\lambda}_{B}^{(i) T} & \dot{\lambda}_{C}^{(i) T}\end{array}\right]^{T}$ is a vector of $3 m$ elements containing the plastic multiplier rates $\dot{\lambda}_{J}^{(i)}$ defined in correspondence of three corners $(A, B, C)$ of a rectangular subcell, whereas $\mathbf{A}_{U(i)}^{\mathrm{eq}}$ and $\mathbf{A}_{\lambda(i)}^{\mathrm{eq}}$ are, respectively, $9 \times 7$ and $9 \times 3 m$ matrices. Thus, plastic admissibility equations for each cell can be assembled, giving rise to a global constraint equation

$$
\mathbf{A}_{U}^{\mathrm{eq}} \mathbf{U}+\mathbf{A}_{\lambda}^{\mathrm{eq}} \dot{\lambda}=\mathbf{0}
$$

where $\mathbf{A}_{U}^{\mathrm{eq}}=\left[\begin{array}{lll}\mathbf{A}_{U(1)}^{\mathrm{eq} T} & \ldots & \mathbf{A}_{U(6)}^{\mathrm{eq} T}\end{array}\right]^{T} ; \dot{\boldsymbol{\lambda}}=\left[\begin{array}{lll}\dot{\boldsymbol{\lambda}}^{(1) T} & \ldots & \dot{\boldsymbol{\lambda}}^{(6) T}\end{array}\right]^{T} ;$ and $\mathbf{A}_{\lambda}^{\mathrm{eq}}=$ following $(6 \cdot 9) \times(6 \cdot 3 m)$ matrix:

$$
\mathbf{A}_{\lambda}^{\mathrm{eq}}=\mathbf{A}_{\lambda(1)}^{\mathrm{eq}} \oplus \mathbf{A}_{\lambda(2)}^{\mathrm{eq}} \oplus \cdots \oplus \mathbf{A}_{\lambda(6)}^{\mathrm{eq}}
$$

where denotes the direct sum. Let $B C$ be the diagonal of the $i$ th subcell running through the vertices $B$ and $C$. Internal dissipated power on the given $i$ th subcell with area $\Omega^{(i)}$ can be expressed as

$$
\pi_{\text {in }}^{(i)}=\frac{\boldsymbol{\Omega}^{(i)}}{2}\left(\mathbf{b}_{i n}^{(i) T} \dot{\boldsymbol{\lambda}}_{B}^{(i)}+\mathbf{b}_{i n}^{(i) T} \dot{\boldsymbol{\lambda}}_{C}^{(i)}\right)=\frac{\boldsymbol{\Omega}^{(i)}}{2}\left[\begin{array}{lll}
\mathbf{0}_{1 \times m} & \mathbf{b}_{i n}^{(i) T} & \mathbf{b}_{i n}^{(i) T}
\end{array}\right] \dot{\boldsymbol{\lambda}}^{(i)}
$$

where $\mathbf{0}_{1 \times m}=$ vector of $m$ null elements. Now, it is possible to compute the overall power dissipated within the RVE as a whole by summation of the power dissipated within each subcell

$$
\pi_{\text {in }}=\sum_{i=1}^{6} \frac{\boldsymbol{\Omega}^{(i)}}{2}\left[\begin{array}{lll}
\mathbf{0}_{1 \times m} & \mathbf{b}_{i n}^{(i) T} & \mathbf{b}_{\text {in }}^{(i) T}
\end{array}\right] \dot{\lambda}^{(i)}
$$

Then, a vector of the macroscopic stress components is defined as $\boldsymbol{\Sigma}=\Lambda\left[\begin{array}{lll}\alpha & \beta & \gamma\end{array}\right]^{T}$, in which the symbols denote the load multiplier $\Lambda$ and the director cosines $(\alpha, \beta, \gamma)$ of the direction of the 
macroscopic stress $\boldsymbol{\Sigma}$ within the space of homogenized stresses. External load power can be simply computed as $\pi_{e x}=\Lambda\left[\begin{array}{lll}\alpha & \beta & \gamma\end{array}\right] \mathbf{D}$ coupled with the normalization condition $\left[\begin{array}{ccc}\alpha & \beta & \gamma\end{array}\right] \mathbf{D}=1$. Finally, the homogenized yield surface can be obtained point by point by solving the constrained optimization problem written as follows:

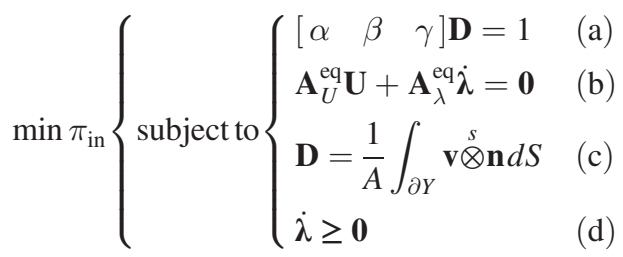

in which the equations have the following meaning: normalization condition (a), admissibility of the plastic flow (b), homogenized strain rate to local velocity field relation (c), and nonnegativity of plastic multipliers (d).

The decision variables in the minimization problem in Eq. (23) are the macroscopic strain rate $\mathbf{D}$ (three components), the seven DOFs representing the microscopic field of velocity, and the $6 \times$ $3 m$ plastic multipliers $\dot{\lambda}$. The normalization condition ultimately allows writing $\Lambda=\min \pi_{\text {in }}$.

With the iterative solution of Eq. (23), it is possible to easily provide a linearization for $f(\boldsymbol{\sigma})$, the assigned yield surface, given the $i$ th plane representing $f(\boldsymbol{\sigma})$, indicated by the equation $A_{i} \sigma_{n n}+$ $B_{i} \sigma_{n s}+C_{i} \sigma_{n t}=1$. Thus, Eq. (14) simplifies to the equation

$$
\Delta \tilde{\mathbf{u}}=\left[\begin{array}{c}
\sum_{i=1}^{N^{p l}} A_{i} \dot{\lambda}^{i} \\
\sum_{i=1}^{N^{p l}} B_{i} \dot{\lambda}^{i} \\
\sum_{i=1}^{N^{p l}} C_{i} \dot{\lambda}^{i}
\end{array}\right]
$$

where $\dot{\lambda}^{i}=i$ th plane plastic multiplier; and $N^{p l}=$ total number of linearization planes used.

The previous constraint must hold for each point $P_{i}$ of each interface. Because for each point of each interface, a set of $N^{p l}$ unknown plastic multipliers is defined, the total number of unknown plastic multipliers is equal to $N^{p l}\left(N_{s d}+1\right) 2 N_{I}$.

On each interface $i$ covering the surface $S_{i}$, the internal dissipated power is defined as the integral

$$
P_{\mathrm{int}, i}^{M-M}=\int_{S_{i}} \boldsymbol{\sigma} \cdot \Delta \tilde{\mathbf{u}} d S
$$

in the local reference system. Such an integral is evaluated through a Gauss quadrature method, as described in the previous section.

\section{Power Dissipation on FRP-FRP Interfaces}

FRP NURBS elements are assumed to be infinitely rigid, as are masonry NURBS elements. Hence, plastic dissipation is permitted along interfaces between adjoining elements only, and is produced as a consequence of longitudinal stresses in the direction of fibers. To enforce plastic compatibility along FRP-FRP interfaces and correctly evaluate dissipation power, the midline of each interface has been subdivided into an assigned number $\left(N_{s d}^{F}+1\right)$ of points $P_{i}$ [Fig. 4(b)]. On each point $P_{i}$, a local reference system $\left(\mathbf{n}^{F}, \mathbf{s}^{F}, \mathbf{t}^{F}\right)$ has been defined, where $\mathbf{n}^{F}$ is the unit vector normal to the interface (and parallel to the fiber direction), $\mathbf{s}^{F}$ is the tangential unit vector in the longitudinal direction, and $\mathbf{t}^{F}$ is the tangential unit vector in the transversal direction. Continuity of the field of velocity is enforced at interfaces between adjoining FRP elements along directions $\mathbf{S}^{F}$ and $\mathbf{t}^{F}$ only, whereas velocity jumps are allowed along direction $\mathbf{n}^{F}$. Normally, because of their negligible thickness, FRP strips buckle under the action of even the lowest compressive stresses. To this extent, different tension and compression limit stresses are imposed, respectively, $f_{\text {FRP }}^{+}$[equal to $f_{\text {fdd }}$ or $f_{\text {fdd,rid }}$ according to CNR-DT200 (2013); see the details in the subsequent section] for crisis in tension and $f_{\mathrm{FRP}}^{-} \approx 0$ for buckling in compression.

To abide by kinematic admissibility, for every jump in velocity at an interface, the following equality constraint, which particularizes the associated flow rule in Eq. (14), must hold:

$$
\Delta \tilde{\mathbf{u}}=\left[\begin{array}{c}
\Delta \tilde{u}_{n} \\
\Delta \tilde{u}_{t} \\
\Delta \tilde{u}_{s}
\end{array}\right]=\left[\begin{array}{c}
\lambda_{i}^{I-\mathrm{FRP}+}-\lambda_{i}^{I-\mathrm{FRP}-} \\
0 \\
0
\end{array}\right]
$$

where $\lambda_{i}^{I-\mathrm{FRP}+}$ and $\lambda_{i}^{I-\mathrm{FRP}-}=$ plastic multipliers at point $P_{i}$ on the FRP-FRP interface corresponding to $f_{\text {FRP }}^{+}$and $f_{\text {FRP }}^{-}$, respectively. Because for each point of each FRP-FRP interface a set of two unknown plastic multipliers is defined, the total number of unknown plastic multipliers for FRP-FRP interfaces is equal to $2\left(N_{s d}^{F}+1\right) N_{I}^{F-F}$. On the other hand, the power dissipated along the $i$ th FRP-FRP interface may be easily evaluated as

$$
P_{\mathrm{int}, i}^{F-F}=s \int_{L_{i}} \boldsymbol{\sigma} \cdot \Delta \tilde{\mathbf{u}} d l=s \int_{L_{i}}\left(f_{\mathrm{FRP}}^{+} \lambda_{i}^{I-\mathrm{FRP}+}+f_{\mathrm{FRP}}^{-} \lambda_{i}^{I-\mathrm{FRP}-}\right) d l
$$

where $s=$ thickness of the interface (i.e., of the FRP strip); and $L_{i}=$ midline of the FRP-FRP interface considered.

\section{Power Dissipation on FRP-Masonry Interfaces}

Adhesion between the reinforcement and the underlying material represents a paramount aspect when considering the use of fiberreinforced composites for retrofitting masonry structures. Indeed, delamination is a very complex phenomenon that brings considerable issues to the structural analysis problem because it depends on a considerable number of parameters and affects materials having different mechanical properties (fibers, brickwork, and glue), and this is particularly true when using limit analysis. Many experimental works in the literature have proved that the delamination crisis is governed by failure in masonry (Ceroni et al. 2003). In other words, upon delamination, the debonding FRP strip peels masonry material, taking away a nonnegligible portion of brickwork.

Today, many codes exist [see, for instance, the Italian code CNR-DT200 (2013)] that allow assessment of delamination of FRP strips from their support by using simple but sound mathematical relations. To keep things simple, in the following discussion, the authors adopt the suggestions contained within the Italian technical norm (CNR-DT200 2013).

In particular, the delamination phenomenon is taken into account through a conventional approach, which consists of adequately limiting the traction force acting within the FRP strip. More precisely, if the bond length $l_{b}$ is greater than the optimal bond length $l_{e}$, the design tensile strength of a FRP strip $f_{\text {fdd }}$, which has been used in a previous subsection, is given by the relation

$$
f_{\mathrm{fdd}}=\frac{1}{\gamma_{f d} \sqrt{\gamma_{M}}} \sqrt{\frac{2 \cdot E_{\mathrm{FRP}} \cdot \Gamma_{F k}}{t_{\mathrm{FRP}}}}
$$

where case $l_{b} \leq l_{e} f_{\mathrm{fdd}}$ is given by the following:

$$
f_{\mathrm{fdd}, \mathrm{rid}}=f_{\mathrm{fdd}} \frac{l_{b}}{l_{e}}\left(2-\frac{l_{b}}{l_{e}}\right)
$$

In Eqs. (28) and (29), $f_{\text {fdd }}$ and $f_{\text {fdd,rid }}=$ design bond strength and the reduced design bond strength, respectively; $E_{\mathrm{FRP}}=$ elasticity modulus of FRP; $t_{\mathrm{FRP}}=$ FRP thickness; $\gamma_{f d}=$ safety factor equal 
to $1.20 ; \gamma_{M}=$ partial safety factor for masonry material, which in this case is assumed to be equal to $1.0 ; l_{b}=$ bond length of FRP strips; and $l_{e}=\sqrt{E_{\mathrm{FRP}} \cdot t_{\mathrm{FRP}} / 2 \cdot f_{\mathrm{mtm}}}=$ optimal bond length, equal to the smallest bond length capable of sustaining the maximum anchoring force. The quantity $\Gamma_{F k}$ in Eq. (28) describes the specific fracture energy of the FRP-reinforced masonry upon delamination. At this point, it should be observed that correction of the specific fracture energy is still an open issue, mainly because masonry mechanical properties are widely variable.

Nevertheless, as shown in Fig. 6(b), the Italian norm suggests a particular $\tau_{b}$-slip constitutive law, which permits indirect estimation of the shear limit stress $f_{b}$ to be used for FRP-masonry interfaces once that ultimate slip is known (usually, the ultimate slip is assumed to be equal to $0.2 \mathrm{~mm}$ ).

From results contained in the literature, it could be inferred that a damaging material model would be a better choice when trying to assess the mechanical response of FRP-reinforced masonry structures (Luciano and Sacco 1998; Marfia and Sacco 2001). Such a choice, in principle, would impede the use of limit analysis, which, as is well known, relies on infinite material ductility and perfect plasticity assumptions. Nevertheless, in agreement with suggestions contained in CNR-DT200 (2013), limit analysis is still a very useful tool to get a quick and reliable evaluation of the load-bearing capacity of a given masonry structure. In fact, the code (CNRDT200 2013) recognizes that delamination of FRP strips is usually a brittle phenomenon. However, it suggests evaluating the effectiveness of the reinforcement by assessing the inhibition of the failure mechanism that would have deployed in the unreinforced case once the designer has ruled out FRP delamination through a conventional approach (i.e., by suitably reducing design tensile strength of composite fibers). The subsequent change in the failure mechanism, according to the upper bound theorem, reveals an increase in the bearing capacity of the structure. In the Italian building code (NTC 2008) and the related explicative instructions (CIRC 2009), the upper bound theorem of limit analysis is the tool of choice for the kinematic analysis of a priori-determined failure mechanisms in masonry constructions, under the hypothesis of negligible tensile strength. Moreover, other authors have successfully investigated the capabilities of limit analysis for the assessment of FRP-reinforced masonry structures (e.g., Ascione et al. 2005; Caporale et al. 2006).

As previously mentioned, the principal consequence of a given retrofitting intervention through the use of FRP strips is, in fact, to impede the triggering of the collapse mechanism causing failure in the unreinforced conditions with the consequent generation of a different failure mechanism with a greater internal dissipated power and a resulting higher collapse multiplier. Because "hand" calculations cannot be easily carried out for general curved masonry structures with arbitrary loading, an upper bound procedure, such the one here presented, is especially suited for a fast and computationally inexpensive evaluation of the load-bearing capacity of general FRP-reinforced masonry vaults.

As previously anticipated, the FRP-masonry interface is a NURBS surface belonging to the extrados or intrados of the vaulted surface.

To take into account dissipation along the FRP-masonry interface, an assigned number $N_{P}^{M-F}$ of Gauss points have been fixed on the surface. In each of these points, a local reference system $\left(\mathbf{s}^{I}, \mathbf{t}^{I}, \mathbf{n}^{I}\right)$ is defined, where $\mathbf{s}^{I}$ is the unit vector tangential to the surface in the direction of fibers, $\mathbf{t}^{I}$ is the tangential unit vector in the transversal direction, and $\mathbf{n}^{I}$ is the unit vector normal to the interface. In each point, the vector field $\boldsymbol{\sigma}=\left(\tau_{s}, \tau_{t}, \sigma_{n}\right)$ representing the stresses acting along the local axes $\left(\mathbf{s}^{I}, \mathbf{t}^{I}, \mathbf{n}^{I}\right)$ can be introduced as well.

Again, to apply limit analysis theorems, jumps in the field of velocity at the FRP-masonry interfaces must be kinematically admissible and, therefore, an associate flow rule must hold. As for masonry-masonry interfaces, the failure surface for FRP-masonry interfaces can be easily linearized as $A_{k} \tau_{s i}+B_{k} \tau_{t i}+C_{k} \sigma_{n i}=D_{k}$, $k=1, \ldots, N_{P L}^{M-F}$ (where $N_{P L}^{M-F}$ is the number of planes used for linearizing the surface). In this case, the flow rule in Eq. (14) is particularized as follows:

$$
\left[\mathbf{u}_{i}\right]=\left[\begin{array}{c}
\Delta u_{s}^{i} \\
\Delta u_{t}^{i} \\
\Delta u_{n}^{i}
\end{array}\right]=\left[\begin{array}{ll}
\sum_{k=1}^{N_{P L}^{M-F}} & A_{k} \dot{\lambda}_{i}^{M-F, k} \\
\sum_{k=1}^{N_{P L}^{M-F}} & B_{k} \dot{\lambda}_{i}^{M-F, k} \\
\sum_{k=1}^{N_{P L}^{M-F}} & C_{k} \dot{\lambda}_{i}^{M-F, k}
\end{array}\right]
$$

Eq. (30) constitutes additional equality constraints on the problem, in which $i=A, B, C$, whereas $\dot{\lambda}_{i}^{M-F, k}$ is the $k$ th plastic multiplier rate relative to the $k$ th linearizing plane. The Italian design code for FRP reinforcement suggests specific $\sigma-\tau_{s}-\tau_{t}$ failure surfaces for FRP-masonry interfaces, as depicted in Fig. 6(b), in which $f_{b}$ is the interface shear strength and $f_{m t}$ describes masonry tensile strength.

An important body of experimental work clearly illustrated that strip delamination along curved structural surfaces always happens in the presence of nonnegligible normal forces between the support and the reinforcing element. Therefore, estimating the actual strength of the interfaces is crucial. It may be of interest to observe that interface strength with respect to normal stresses is here considered equal for both tension and compression. In a more sophisticated model, different limiting strengths for tensile and

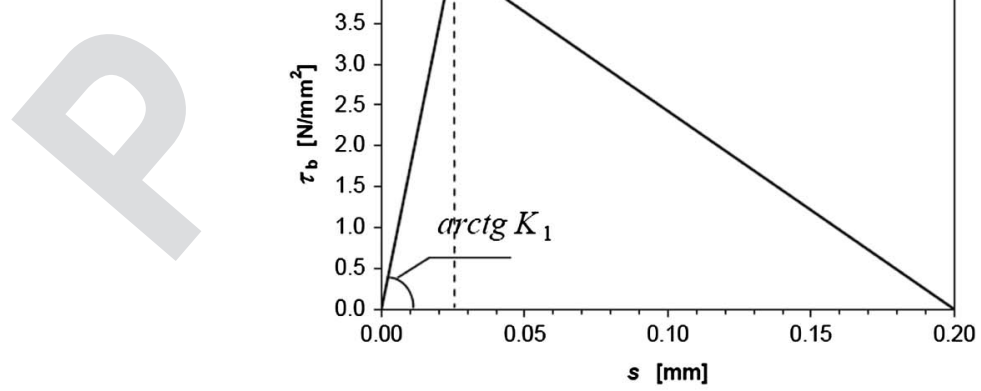

(a)

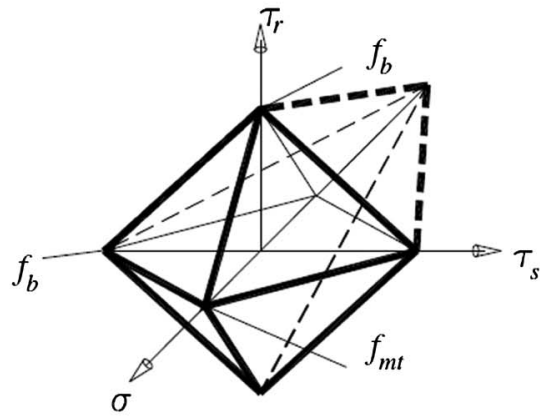

(b)

Fig. 6. (a) $\tau_{b}$-slip constitutive law; (b) $\sigma-\tau_{s}-\tau_{t}$ failure surfaces for FRP-masonry interfaces adopted 
compressive behavior should be assumed, given that $f_{m t}$ is closely connected to the tensile and compression strength of bricks. In the present model, any nonlinear failure surface for the FRP-masonry bond could be included without any additional complication.

Because for each point of each FRP-masonry interface a set of $N_{P L}^{M-F}$ unknown plastic multipliers is defined, the total number of unknown plastic multipliers for FRP-FRP interfaces is equal to $N_{P L}^{M-F} N_{P}^{M-F} N_{I}^{M-F}$.

Again, on each FRP-masonry interface $i$ covering the surface $S_{i}$, the internal dissipated power is defined as the integral

$$
P_{\mathrm{int}, i}^{M-M}=\int_{S_{i}} \boldsymbol{\sigma} \cdot \Delta \tilde{\mathbf{u}} d S
$$

in the local reference system, where both $\boldsymbol{\sigma}$ and $\Delta \tilde{\mathbf{u}}$ have been defined in the present subsection. Such an integral is evaluated through a Gauss quadrature method, as described in the previous section.

\section{Nonnegativity of Plastic Multipliers and Normalization Condition}

An additional constraint that must be included in the linear programming problem is the nonnegativity of each plastic multiplier

$$
\dot{\lambda}_{i j} \geq 0
$$

The last condition to be applied is the so-called normality condition, which requires that the external power dissipated by the live load $\mathbf{1} \cdot \Gamma$ set equal to one is itself equal to one; i.e.

$$
P_{\Gamma=1}=1
$$

This condition allows Eq. (10) to be rewritten in the following way:

$$
\Gamma=\sum_{i=1}^{N_{I}^{\mathrm{TOT}}} P_{\mathrm{int}}^{i}-P_{F_{0}}
$$

\section{Linear Programming Problem}

Remembering Eq. (34) and following the kinematic theorem of limit analysis, the related LP problem can be stated as follows:

$$
\min \left\{\sum_{i=1}^{N_{I}} P_{\text {int }}^{i}-P_{\mathbf{F}_{0}}\right\}
$$

under the geometric constraints in Eq. (13); the compatibility constraints in Eqs. (24), (26), and (30); the nonnegativity of plastic multiplier constraints in Eq. (32); and the normality condition in Eq. (33). The unknowns of the LP problem are the $6 \cdot N_{E}$ generalized velocity components of the center of mass of each element and the total number of plastic multipliers at each point of each interface.

\section{Genetic Algorithm Mesh Adjustment}

Because dissipation is allowed only along interfaces, it is necessary to introduce an algorithm that allows adjustment of the mesh to find the minimum collapse multiplier among all possible configurations and therefore to determine the actual collapse mechanism.

Relying on a process that mimics evolution in a population through natural selection, GAs allow solving of both constrained and unconstrained optimization problems. Such algorithms iteratively adjust a population in which each individual corresponds to a candidate solution of the problem. After each iteration, the GA selects individuals in a random way, picking them from the current generation, to establish parents for the next generation of offspring. After each generation, the population "evolves" toward an optimal solution.

A NURBS mesh of a vaulted surface is determined by a given number $N_{\text {par }}$ of real parameters $p_{1}, p_{2}, \ldots, p_{N p a r}$ that depend on the type of collapse mechanism that must be detected. A given NURBS mesh is regarded as an individual, and each individual is written as an array with $1 \times N_{\text {par }}$ elements

$$
\text { Individual }=\left[p_{1}, p_{2}, \ldots, p_{N \text { par }}\right]
$$

The cost of each individual is evaluated by means of a cost function $f$ at the parameters $p_{1}, p_{2}, \ldots, p_{N \text { par. }}$. The function $f$ is defined as a function that outputs the collapse load multiplier $\lambda_{c}$ for every assigned individual (i.e., an assigned mesh on the surface) through the implementation of the limit analysis procedure described in the "Kinematic Limit Analysis" section

$$
\lambda_{c}=f(\text { individual })=f\left(p_{1}, p_{2}, \ldots, p_{N \text { par }}\right)
$$

To initialize the GA, an initial population of $N_{i \text { pop }}$ individuals is defined. The whole population is represented by a matrix in which each row is a $1 \times N_{\text {par }}$ array (i.e., the individual) of continuous parameters. Starting from the initial population constituted by $N_{i \text { pop }}$ individuals, a complete matrix of $N_{i \text { pop }} \times N_{\text {par }}$ random entries can be generated as follows:

$$
\mathrm{IPOP}=(h i-l o) \times \operatorname{random}\left\{N_{i \mathrm{pop}}, N_{\text {par }}\right\}+l o
$$

where $\operatorname{random}\left\{N_{i \text { pop }}, N_{\text {par }}\right\}=$ function generating an $N_{\text {ipop }} \times N_{\text {par }}$ matrix of uniformly randomized numbers; and $h i$ and $l o=$ highest and lowest values in the range of parameters. As anticipated previously, individuals are not all equal: the cost function assesses how much each individual is worth.

To establish chromosomes belonging to the initial population that are suited to survive and produce offspring for the next generation, the cost of the $N_{i \text { pop }}$ individuals is ranked from the lowest to the highest. The best $N_{\text {pop }}$ individuals for the next algorithmic iteration are retained, and the rest are left to die. This process is called natural selection, and from this point on, the size of the population at each generation is $N_{\text {pop }}$. Other more sophisticated selection operators have been introduced in the literature (Goldberg 1989; Kwon et al. 2003) as, for example, proportional and tournament selection operators.

Then, an equal number of mothers and fathers is selected within the $N_{\text {pop }}$ individuals, which pair in some random fashion. There are various reasonable ways to pair individuals. In this paper, a weighted cost selection with assigned probabilities is used (Haupt and Haupt 2004). Each pair of individuals generates two offspring containing traits belonging to each of the parents. Mating is carried out by choosing an assigned number of crossover points (at least one) on the chromosome. The parameters between these points are simply swapped between the parents. In this paper, a crossover operator with multiple points is used. The crossover points $k_{i}=$ $[1,2, \ldots, c-1]$ are selected in a random way for each pair of two individuals, represented by $c$ chromosomes. Moreover, care must be taken to prevent the GA from converging too fast into one given region of the cost surface. Indeed, this may be not good if the problem being modeled has several local minima in which the solution may get trapped. To circumvent issues related to exceedingly fast convergence, the algorithm is forced to test other regions of the cost surface by adding random changes, known as mutations, for a given share of parameters. A classic mutation operator is applied to all $N_{\text {pop }}$ individuals at each generation. For each individual $\mathbf{p}_{i}$, the 


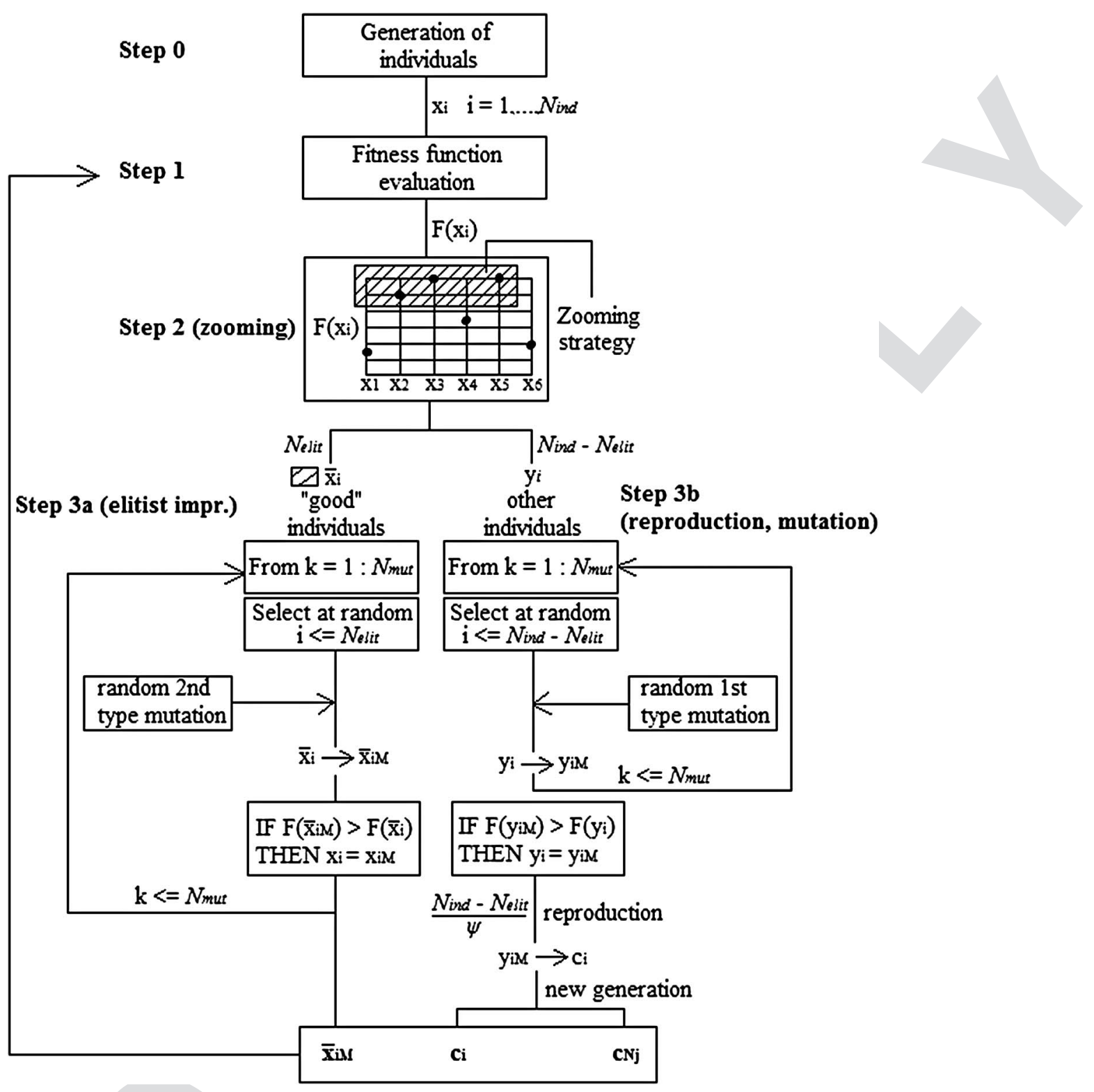

Fig. 7. Pseudocode for the adopted GA

mutation operator acts in a stochastic way on all the chromosomes of the individual subject to mutation (i.e., randomly changing one of the chromosomes in the individual involved in the process of generating offspring). A mutation probability of $15 \%$ has been chosen in this paper.

The algorithm described can be improved by adding zooming with an elitist strategy (e.g., Milani and Milani 2008) with the aim of considerably enhancing both algorithmic efficiency and robustness. In particular, the zooming approach provides for a subdivision of the initial population into two sets of individuals $\overline{\mathbf{x}}=$ $\left\{\overline{\mathbf{x}}_{i}: i=1, \ldots, N_{\text {elit }}\right\}$ and $\mathbf{y}=\mathbf{x}-\overline{\mathbf{x}}=\left\{\mathbf{y}_{i}: i=1, \ldots, N_{\text {pop }}-N_{\text {elit }}\right\}$, which, for each iteration, collects individuals with the highest fitness into an "elite" subpopulation. The dimension of this subpopulation is defined by the user and is denoted by $N_{\text {elit }}$. Subsequently, to each individual in the set $\overline{\mathbf{x}}$, only a high probability of mutation is assigned (i.e., not a crossover) to improve individual fitness. From a practical viewpoint, zooming must be user defined through the assignment of the commonly named zooming percentage $z \%$, which describes the ratio (in percent) between the sizes $N_{\text {pop }}$ and $N_{\text {elit }}$ of the initial population and the subgroup $\overline{\mathbf{x}}$, respectively. Even though in this paper zooming percentage $z_{\%}$ is assumed to be constant (equal to $5 \%$ ), it can still be suitably reduced when passing from a generation to the successive one by means of an exponential reduction scheme. A logic tree for the algorithm adopted is depicted in Fig. 7.

\section{Conclusions}

A new GA-NURBS-based approach for the kinematic limit analysis of FRP-reinforced masonry vaulted structures has been introduced. The present Part I has been devoted to illustrating and discussing the theoretical framework adopted. The main idea consists of exploiting properties of NURBS functions to develop a computationally efficient adaptive limit analysis procedure that allows quick evaluation of the collapse load multiplier of any given masonry vault starting from its 3D model, which can be obtained with any free form modeler (e.g., Rhinoceros) natively working with NURBS entities. It is therefore possible to bridge the 3D modeling environment, which is very popular among practitioners, with 
a structural limit analysis environment in the most natural way, thus requiring the least effort from the final user and providing a high computational efficiency. More precisely, a given reinforced masonry vault can be geometrically represented by NURBS parametric surfaces, and a NURBS mesh composed of very few elements of the given surface can be generated both for masonry and FRP reinforcement. Even if the number of elements is very low, the mesh still provides an exact representation of the structural geometry, thanks to its NURBS representation. Each element of the mesh is a NURBS surface itself and can be idealized as a rigid body. A homogenized upper bound limit analysis formulation, which takes into account the main characteristics of masonry material and FRP reinforcement, is deduced, with internal dissipation allowed exclusively along element edges. The approach is shown to be able to well predict the load-bearing capacity of any FRP-reinforced masonry vault of arbitrary shape provided that the initial mesh is adaptively adjusted by means of a suitable GA to enforce that element edges accurately approximate the actual failure mechanism. Part II of the present paper is devoted to validating and discussing the proposed approach through a number of numerical simulations. More work examples are synthetized in Chiozzi et al. (2016b).

\section{References}

Aboudi, J. ed. (2013). Mechanics of composite materials: A unified micromechanical approach, Elsevier, Amsterdam, Netherlands.

Aiello, M. A., and Sciolti, S. M. (2006). "Bond analysis of masonry structures strengthened with CFRP sheets." Constr. Build. Mater., 20(1-2), 90-100.

Angelillo, M., Babilio, E., and Fortunato, A. (2013). "Singular stress fields for masonry-like vaults." Contin. Mech. Thermodyn., 25(2), 423-441.

Ascione, L., Feo, L., and Fraternali, F. (2005). "Load carrying capacity of 2D FRP/strengthened masonry structures." Compos. Part B Eng., 36(8), 619-626.

Block, P., Ciblac, T., and Ochsendorf, J. (2006). "Real-time limit analysis of vaulted masonry buildings." Comput. Struct., 84(29-30), $1841-1852$

Block, P., and Lachauer, L. (2013). "Three-dimensional (3D) equilibrium analysis of gothic masonry vaults." Int. J. Archit. Herit., 8(3), 312-335.

Cancelliere, I., Imbimbo, M., and Sacco, E. (2010). "Experimental tests and numerical modeling of reinforced masonry arches." Eng. Struct., 32(3), 776-792.

Caporale, A., Luciano, R., and Rosati, L. (2006). "Limit analysis of masonry arches with externally bonded FRP reinforcements." Comput. Methods Appl. Mech. Eng., 196(1), 247-260.

Ceroni, F., Pecce, M. R., Manfredi, G., and Marcari, G. (2003). "Experimental bond behaviour in masonry elements externally reinforced with FRP laminates." Proc., Int. Conf. on Composites in Construction, Univ. of Calabria, Cosenza, Italy.

Chen, S., Liu, Y., and Cen, Z. (2008). "Lower bound shakedown analysis by using the element free Galerkin method and non-linear programming." Comput. Methods Appl. Mech. Eng., 197(45-48), 3911-3921.

Chiozzi, A., Malagù, M., Tralli, A., and Cazzani, A. (2016a). "ArchNURBS: NURBS-based tool for the structural safety assessment of masonry arches in MATLAB." J. Comput. Civ. Eng., 4015010.

Chiozzi, A., Milani, G., Grillanda, N., and Tralli, A. (2016b). "An adaptive procedure for the limit analysis of FRP reinforced masonry vaults and applications.” Am. J. Eng. Appl. Sci., 9(3), 735-745.

Chiozzi, A., Milani, G., Grillanda, N., and Tralli, A. (2017a). "A fast and general upper-bound limit analysis approach for out-of-plane loaded masonry walls." Meccanica, 1-24.

Chiozzi, A., Milani, G., and Tralli, A. (2016c). "Fast kinematic limit analysis of FRP masonry vaults through a new genetic algorithm NURBS-based approach." Proc., 7th European Congress on Computational Methods in Applied Sciences and Engineering, National Technical Univ. of Athens, Athens, Greece.
Chiozzi, A., Milani, G., and Tralli, A. (2017b). "Fast kinematic limit analysis of FRP-reinforced masonry vaults. II: Numerical simulations." J. Eng. Mech., 10.1061/(ASCE)EM.1943-7889.0001268, XX, x-X.

Chiozzi, A., Milani, G., and Tralli, A. (2017c). "A genetic algorithm NURBS-based new approach for fast kinematic limit analysis of masonry vaults." Comput. Struct., 182, 187-204.

Chiozzi, A., Simoni, M., and Tralli, A. (2016d). "Base isolation of heavy non-structural monolithic objects at the top of a masonry monumental construction." Mater. Struct., 49(6), 2113-2130.

Christiansen, E., and Andersen, K. D. (1999). "Computation of collapse states with von mises type yield condition." Int. J. Numer. Methods Eng., 46(8), 1185-1202.

Christiansen, E., and Pedersen, O. S. (2001). "Automatic mesh refinement in limit analysis." Int. J. Numer. Methods Eng., 50(6), 1331-1346.

CIRC. (2009). "Instructions for the application of the new Italian building code referred to in D.M. 14/01/2008." Gazzetta Ufficiale della Repubblica Italiana, Italy (in Italian).

CNR-DT200. (2013). "Instructions for the design, execution and control of retrofit interventions on buildings by means of FRP composites." Italian Research Council, Italy (in Italian).

Como, M., ed. (2013). Statics of historic masonry construction, Springer, Berlin.

Corradi, M., Borri, A., and Vignoli, A. (2002). "Strengthening techniques tested on masonry structures struck by the Umbria-Marche earthquake of 1997-1998." Constr. Build. Mater., 16(4), 229-239.

Cottrell, J. A., Hughes, T. J. R., and Bazilevs, Y. (2009). Isogeometric analysis: Toward integration of $C A D$ and FEA, Wiley, Chichester, U.K.

Fagone, M., and Briccoli Bati, S. (2008). "Analysis and mechanical characterization of the failure modes of FRP reinforcements applied to masonry bricks." Proc., GIMC Symp., Univ. of Sassari, Sassari, Italy (in Italian).

Fedele, R., and Milani, G. (2011). "Three-dimensional effects induced by FRP-from-masonry delamination." Compos. Struct., 93(7), 1819-1831.

Ghiassi, B., Marcari, G., Oliveira, D. V., and Lourenço, P. B. (2012). "Numerical analysis of bond behavior between masonry bricks and composite materials." Eng. Struct., 43, 210-220.

Goldberg, D. E. (1989). "Genetic algorithms in search, optimization, and machine learning." Addison-Wesley, Boston.

Grande, E., Milani, G., and Sacco, E. (2008). "Modelling and analysis of FRP-strengthened masonry panels." Eng. Struct., 30(7), 1842-1860.

Haupt, R. L., and Haupt, S. E. (2004). Practical genetic algorithms, Wiley, Hoboken, NJ.

Heyman, J. (1997). Stone skeleton: Structural engineering of masonry architecture, Cambridge University Press, Cambridge, U.K.

Huerta, S. (2008). "The analysis of masonry architecture: A historical approach.” Archit. Sci. Rev., 51(6), 297-328.

Korany, Y., Drysdale, R., and Chidiac, S. E. (2001). "Retrofit of non-strengthened masonry buildings: The state of the art." Proc., 9th Canadian Masonry. Symp., Canada Masonry Design Centre, Calgary, Canada, 88-115.

Kwon, Y.-D., Kwon, S.-B., Jin, S.-B., and Kim, J.-Y. (2003). "Convergence enhanced genetic algorithm with successive zooming method for solving continuous optimization problems." Comput. Struct., 81(17), $1715-1725$.

Luciano, R., and Sacco, E. (1998). "Damage of masonry panels reinforced by FRP sheets." Int. J. Solids Struct., 35(15), 1723-1741.

Lyamin, A. V., Krabbenhøft, K., Sloan, S. W., and Hjiaj, M. (2004). “An adaptive algorithm for upper bound limit analysis using discontinuous velocity fields." Proc., European Congress Computational Methods in Applied Sciences and Engineering, Jyväskylä Univ., Jyväskylä, Finland.

Lyamin, A. V., Sloan, S. W., Krabbenhøft, K., and Hjiaj, M. (2005). "Lower bound limit analysis with adaptive remeshing." Int. J. Numer. Methods Eng., 63(14), 1961-1974.

Makrodimopoulos, A., and Martin, C. M. (2007). "Upper bound limit analysis using simplex strain elements and second-order cone programming." Int. J. Numer. Anal. Methods Geomech., 31(6), 835-865. 
Makrodimopoulos, A., and Martin, C. M. (2008). "Upper bound limit analysis using discontinuous quadratic displacement fields." Commun. Numer. Methods Eng., 24(11), 911-927.

Marfia, S., and Sacco, E. (2001). "Modeling of reinforced masonry elements." Int. J. Solids Struct., 38(24-25), 4177-4198.

MATLAB [Computer software]. MathWorks, Natick, MA.

Milani, E., Milani, G., and Tralli, A. (2008). "Limit analysis of masonry vaults by means of curved shell finite elements and homogenization." Int. J. Solids Struct., 45(20), 5258-5288.

Milani, G. (2009). "Homogenized limit analysis of FRP-reinforced masonry walls out-of-plane loaded." Comput. Mech., 43(5), 617-639.

Milani, G. (2015). "Upper bound sequential linear programming mesh adaptation scheme for collapse analysis of masonry vaults." Adv. Eng. Softw., 79, 91-110.

Milani, G., and Lourenço, P. B. (2009). "A discontinuous quasi-upper bound limit analysis approach with sequential linear programming mesh adaptation." Int. J. Mech. Sci., 51(1), 89-104.

Milani, G., and Milani, F. (2008). "Genetic algorithm for the optimization of rubber insulated high voltage power cables production lines." Comput. Chem. Eng., 32(12), 3198-3212.

Milani, G., and Taliercio, A. (2015). "In-plane failure surfaces for masonry with joints of finite thickness estimated by a method of cells-type approach." Comput. Struct., 150, 34-51.

Milani, G., and Tralli, A. (2012). "A simple meso-macro model based on SQP for the non-linear analysis of masonry double curvature structures." Int. J. Solids Struct., 49(5), 808-834.

Ngo, N. S., and Tin-Loi, F. (2007). "Shakedown analysis using the p-adaptive finite element method and linear programming." Eng. Struct., 29(1), 46-56.

NTC. (2008). "New Italian building code." Gazzetta Ufficiale della Repubblica Italiana, Italy (in Italian).
Oliveira, D. V., Basilio, I., and Lourenço, P. B. (2011). "Experimental bond behavior of FRP sheets glued on brick masonry." J. Compos. Constr., 10.1061/(ASCE)CC.1943-5614.0000147, 32-41.

Piegl, L., and Tiller, W. (1995). The NURBS book, Springer, New York. Rhinoceros [Computer software]. Robert McNeel \& Associates, Seattle.

Saadatmanesh, H. (1994). "Fiber composites for new and existing structures." Struct. J., 91(3), 346-354.

Sloan, S. W., and Kleeman, P. W. (1995). "Upper bound limit analysis using discontinuous velocity fields." Comput. Methods Appl. Mech. Eng., 127(1-4), 293-314.

Taliercio, A. (2014). "Closed-form expressions for the macroscopic in-plane elastic and creep coefficients of brick masonry." Int. J. Solids Struct., 51(17), 2949-2963.

Tin-Loi, F., and Ngo, N. S. (2003). "Performance of the p-version finite element method for limit analysis." Int. J. Mech. Sci., 45(6-7), 1149-1166.

Tralli, A., Alessandri, C., and Milani, G. (2014). "Computational methods for masonry vaults: A review of recent results." Open Civ. Eng. J., 8(1), 272-287.

Triantafillou, T. C. (1998). "Composites: A new possibility for the shear strengthening of concrete, masonry and wood." Compos. Sci. Technol., 58(8), 1285-1295.

Triantafillou, T. C., and Fardis, M. N. (1997). "Strengthening of historic masonry structures with composite materials." Mater. Struct., 30(8), 486-496.

USPRO (U.S. Product Data Association). (1996). "Initial Graphics Exchange Specification, IGES 5.3." U.S. Product Data Association, North Charleston, SC.

Valluzzi, M. R., Valdemarca, M., and Modena, C. (2001). "Behavior of brick masonry vaults strengthened by FRP laminates." J. Compos. Constr., 10.1061/(ASCE)1090-0268(2001)5:3(163), 163-169.

Yu, X., and Tin-Loi, F. (2006). "A simple mixed finite element for static limit analysis.” Comput. Struct., 84(29-30), 1906-1917.

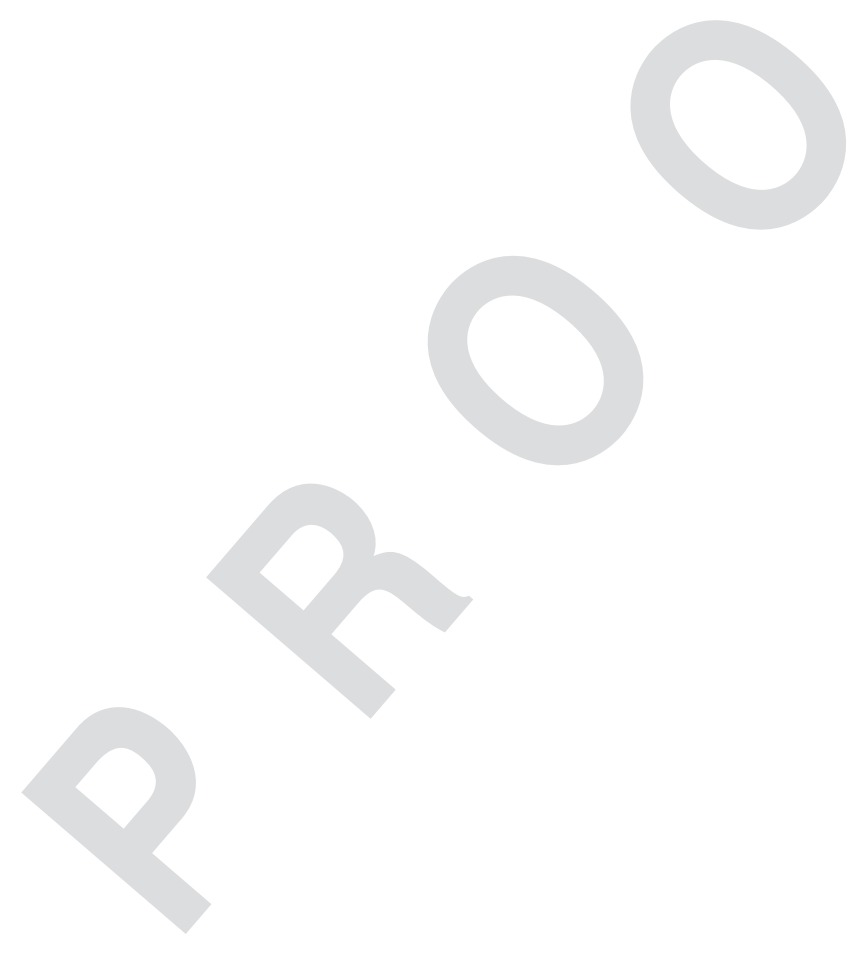

